\title{
Children's Eating Habits, Physical Activity, Sleep, and Media Usage before and during COVID-19 Pandemic in Poland
}

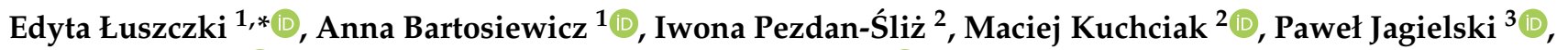 \\ Łukasz Oleksy ${ }^{4}{ }^{\oplus}$, Artur Stolarczyk ${ }^{4}$ and Katarzyna Dereń ${ }^{1} \mathbb{C}$ \\ 1 Institute of Health Sciences, Medical College of Rzeszów University, 35-959 Rzeszów, Poland; \\ abartosiewicz@ur.edu.pl (A.B.); kderen@ur.edu.pl (K.D.) \\ 2 Institute of Physical Culture Sciences, Medical College of Rzeszów University, 35-959 Rzeszów, Poland; \\ ipezdan@ur.edu.pl (I.P.-Ś.); mkuchciak@ur.edu.pl (M.K.) \\ 3 Department of Nutrition and Drug Research, Institute of Public Health, Faculty of Health Sciences, \\ Jagiellonian University Medical College, 31-066 Krakow, Poland; paweljan.jagielski@uj.edu.pl \\ 4 Orthopaedic and Rehabilitation Department, Medical University of Warsaw, 02-091 Warsaw, Poland; \\ loleksy@oleksy-fizjoterapia.pl (Ł.O.); artur.stolarczyk@wum.edu.pl (A.S.) \\ * Correspondence: eluszczki@ur.edu.pl; Tel.: +48-17-851-68-11
}

check for updates

Citation: Łuszczki, E.; Bartosiewicz, A.; Pezdan-Śliż, I.; Kuchciak, M.; Jagielski, P.; Oleksy, Ł.; Stolarczyk, A.; Dereń, K. Children's Eating Habits, Physical Activity, Sleep, and Media Usage before and during COVID-19 Pandemic in Poland. Nutrients 2021 13, 2447. https://doi.org/10.3390/ nu13072447

Academic Editor: Evelyn Parr

Received: 12 June 2021

Accepted: 15 July 2021

Published: 17 July 2021

Publisher's Note: MDPI stays neutral with regard to jurisdictional claims in published maps and institutional affiliations.

Copyright: (c) 2021 by the authors. Licensee MDPI, Basel, Switzerland. This article is an open access article distributed under the terms and conditions of the Creative Commons Attribution (CC BY) license (https:/ / creativecommons.org/licenses/by/ $4.0 /)$.

\begin{abstract}
The COVID-19 pandemic has caused huge changes in people's lifestyle, health, and social relationships. This situation has had an impact on children and adolescents, affecting their health, intellectual, physical, and emotional development. The survey aimed to compare eating behaviors, level of physical activity (PA), hours of sleep, and screen time among Polish children and adolescents aged 6-15 years before and during the COVID-19 pandemic. We obtained self-reported data from 1016 participants at two measurement points before and during the COVID-19 lockdown in Poland to examine the influence of the lockdown and the distance learning on PA, dietary habits, sleep, and media usage of children and adolescents aged 6-15 years. The study identified dietary differences and changes in daily activity patterns (reduced sleep duration with higher sleep quality and reduced physical activity). Additionally, the increase in general media usage was observed during the pandemic alongside a reduction in smartphone usage. Together, the findings indicate increased sleep, physical activity, and reduced media usage and screen time among Polish children and adolescents during the COVID-19 pandemic.
\end{abstract}

Keywords: children and adolescents; COVID-19 pandemic; dietary patterns; media usage; physical activity; sleep

\section{Introduction}

The COVID-19 pandemic has caused huge changes in people's lifestyle, health, and social relationships. The worldwide lockdown has affected socioeconomics, education, health care, and mental illness [1-3]. The situation has had a large influence on children and adolescents, impacting their health, mental, physical, and emotional development [4]. Many children and adolescents in the developed world were already sedentary before the pandemic, spending a lot of time using various technical devices. As reported by Lancet, $81 \%$ of students aged 11-17 were not physically active enough before the pandemic [5]. However, the demands of everyday life were a mobilization to leave home, go to school, meet with friends, and participate in various activities outside the classroom. Due to the pandemic situation and the need to avoid infection, the previous activities were completely reduced, and home quarantine was conducive to a significant increase in computer time. Children and young people faced with the pandemic experienced such enormous changes in their lives as never before. The COVID-19 pandemic has led to rapid, unprecedented changes in the lives of billions of children and adolescents around the world [6,7]. Almost $90 \%$ of children and adolescents, approximated as 1.5 billion youth worldwide, have 
been obliged to stay at home. Apart from studying at school, children undertake many additional activities, such as horse riding, swimming, dancing, learning foreign languages, etc. The limitation of these activities also limited contact with friends, which significantly increased efforts to maintain relationships through social media and various types of communicators [8]. It significantly influenced their health, including nutrition, physical exercise, sleep, and social functioning, increasing their media use [9].

Governments in Europe responded with various measures aimed to slow down the transmission of COVID-19. Those restrictions, which included the closure of schools, playgrounds, and parks, significantly reduced chances for children and adolescents to maintain a healthy lifestyle with physical activity (PA). Children lost opportunities for physical activity in school and outside of school. Recommendations for children and adolescents indicate that they should accumulate $>60$ min of moderate to vigorous-intensity physical activity and involve $<2 \mathrm{~h}$ of screen time/day [10]. Results from countries such as Canada, the U.S., and China demonstrated a reduction in physical activity and rise in screen time during the first wave of the COVID-19 pandemic [11-14]. In another study, only $<10 \%$ of children 5-17 years have achieved physical activity recommendations [15]. Nevertheless, there is a lack of knowledge about the impact in Europe, including Poland.

The first confirmed case in Poland was diagnosed on 4 March 2020. An epidemic emergency state was professed on 20 March pursuant to a Regulation of the Minister of Health [16]. Control measures were implemented on 10-12 March, cancelling mass events and closing kindergartens, schools, universities, and offices; then, they were strengthened on 25 March, limiting religious gatherings and forbidding non-essential travel.

The literature suggests that social restrictions required to lessen the spread of COVID19 have increased inactive behaviors [17], deteriorated sleep patterns [18], and decreased physical activity [19]. Children and adolescents had to make a lot of changes in their everyday lives, including home confinement, school closures, and social distancing rules [20]. The closure of sport clubs as well lower physical activity and staying at home for long hours may result in body mass increase in children and obesity development. However, in developing countries such as Poland, the opposite may appear: the economic aspect of COVID-19 may decrease parents' abilities to provide food supplies for their children and can lead to malnutrition [21]. Additionally, they do not receive meals at school during the lockdown, which can increase the occurrence of malnutrition [22]. Therefore, the hypothesis was that the COVID-19 pandemic caused undernutrition in low and low-medium income countries, especially in children [23].

However, results from the online survey including 1097 people in Poland showed that $43 \%$ of people ate more, while $52 \%$ ate more snacks during the nationwide quarantine. Almost $30 \%$ of adults reported weight gain [24].

Sedentary behavior is a risk factor for cardiometabolic disease also in adults and children [25]. Guidelines recommend $\leq 2 \mathrm{~h}$ of recreational screen time/day for 5-17-yearolds [26]. However, almost $50 \%$ of children in the U.S. aged 6-11 spent $>2 \mathrm{~h} /$ per day on sedentary behavior such as playing video games, watching TV, and using smartphones before the pandemic [27]. Similar results were observed in Poland [28]. Data from studies conducted during the COVID-19 pandemic presented significant (20-66\%) increases in media usage [29].

Sleep disturbances may have an impact on health, attention span, and immune function [30]. For children 6-15 years old, $9-11 \mathrm{~h}$ of uninterrupted sleep are recommended [26]. Moore et al. showed those children were sleeping more hours compared to the period before the COVID-19 pandemic [12]. Likewise, Pietrobelli et al. reported that hours of sleep have risen by $0.65 \mathrm{~h}$ per day during the pandemic compared to the year 2019 in Italian children [13]. However, sleep quality has not been studied within the context of the COVID-19 pandemic among children and adolescents.

To understand the effects of the COVID-19 pandemic on children's and adolescent's nutrition, physical activity, sleep, and media use, we administered an online survey one year after our previous study conducted before the pandemic. The survey aimed to compare 
eating behaviors, level of physical activity, hours of sleep, and screen time among Polish children and adolescents aged 6-15 years before and during the COVID-19 pandemic.

\section{Materials and Methods}

\subsection{Study Design and Sample}

This is a cross-sectional study that was conducted among 1016 participants using a survey technique. The self-reported data were carried out at two measurement points before (pre) and during (peri) the COVID-19 lockdown in Poland to examine the effect of the lockdown and the distance learning on PA, dietary habits, sleep, and media usage of children and adolescents aged 6-15 years.

This study consisted of two parts (pre and peri). The results of the first study have been published [31]. The part before the pandemic was conducted at the turn of February and March 2020 in the randomly selected schools in the Podkarpackie Voivodeship in Poland. The following inclusion criteria for the study were used: aged 6-15 years, the attendance of one of the selected schools, and parents' acceptance of their children's participation in the study. Currently, Poland has an eight-year education system in primary schools for children aged from 6 to 15 years.

The pre-study group consisted of 376 pupils (187 boys and 189 girls) aged 6 to 15 years and was a representative sample of the population of southeast Poland. The study methodology has been published in detail [31].

Assuming a 5\% error threshold and a test power of 0.95 for this population size, the number of children included in the pre-pandemic study was sufficient. In the second measurements point (peri) carried out during the pandemic, by increasing the size of the group to 641 participants, it was possible to reduce the error threshold to $4 \%$, i.e., the test power was 0.96 . The number of children participating in the study is representative of the population of children from the southeastern region of Poland.

One year later, from 1st February until 2nd March 2021 during the COVID-19 pandemic and lockdown in Poland, the second part of the study was conducted. The same methodology with the same questionnaire was used. Target recruitment was a minimum of 376 children collected via convenience sampling in the same part of southeast Poland (Podkarpackie Voivodeship). Children in the defined age group who resided in the region were eligible to participate. After obtaining the consent of the directors of all primary schools and the mayor of the randomly selected city for conducting the research, we sent the information to the children and their parents/guardians via the electronic journal. The message contained information about the planned research and a consent form for parents/guardians for their child to participate in the study. The participants were assured of their voluntary participation and of the anonymity of the study. The total number of the study group during the pandemic was 641 .

\subsection{Weight, Height, and BMI}

In the pre-study, the height was measured three times with an accuracy of $0.1 \mathrm{~cm}$ by means of a Seca 213 portable stadiometer, and the weight was measured assessed with an accuracy of $0.1 \mathrm{~kg}$ using a body composition analyzer (BC-420, Tanita, Tokyo, Japan) [31]. In the peri study, weight and height were declared by the person who filled in the questionnaire during the COVID-19 pandemic.

BMI was calculated as a ratio of weight to height $\left(\mathrm{kg} / \mathrm{m}^{2}\right)$. Centile grids for BMI according to age, sex, and height developed under the project "Developing standards of blood pressure in children and adolescents in Poland, OLAF" were used in our study to define childhood underweight, normal weight, overweight, and obesity [32].

\subsection{Questionnaire Data}

The questionnaires for younger children (6-12 years old) were completed by their parents/guardians, while the adolescents (13-15 years) filled in the online questionnaire on their own. A personal survey link was sent to each parent or/and child. The responses 
of the study participants were automatically stored on the hard drive of the company computer and on a mass storage media. The questionnaires received were checked at the survey center for completeness, and missing questions were added by conducting an interview. There were two additional interviews with participants, which lasted about $15 \mathrm{~min}$. The questionnaire used in the study was divided into four sections and covered the information referring to the children's lifestyle, eating and sleeping habits, the use of technical devices and the Internet, and socio-demographic data. The time needed to complete the questionnaire was approximately $25 \mathrm{~min}$. An example of the questionnaire is attached as a supplementary file. After completing the research, all the data were deposited in the Rzeszow University repository.

To assess the nutrition habits of the respondents, the modified Food Frequency Questionnaire (FFQ-6) was used [33]. Next, questions were connected to the child's sleeping behavior: the amount of time spent sleeping at night and during the day and sleep quality. There were questions: What is the amount of time you sleep during a $24 \mathrm{~h}$ period on school days? What is the amount of time you sleep during a $24 \mathrm{~h}$ period on weekends? During the past month, how would you rate your sleep quality overall? (Possible answers were: Very good, Fairly good, Fairly bad, Very bad). The third part of questionnaire was related to the use of technical devices and the Internet by children [34]. Physical activity was assessed with the question: Over the last week, how many days have you performed $60 \mathrm{~min}$ or more of physical activity that increased your breathing rate? This may include doing sport (football, volleyball, basketball etc.), exercises, brisk walking, or cycling for recreational purposes or on the way to these places. The possible answers were from 0 to 7 days. Details about the questionnaire were described in a previously published study [31].

\subsection{Statistical Analysis}

Results of the study were developed using descriptive statistics: number $(\mathrm{N})$, percentage (\%), mean $(\bar{x})$, median (Me), and standard deviation (SD). The Shapiro-Wilk test was performed to check the normality of the data. A non-parametric Mann-Whitney U test was used. The analysis of variables having the character of qualitative data was carried out with the Pearson chi-square test. The statistical analyses were performed using PS MAGO PRO 6.0 (IBM SPSS STATISTICS 26). Statistical significance was set at $p<0.05 .3$. Results

\section{Results}

Characteristics of the Study Group, Physical Activity, and Sleep

The study was conducted among 1,016 children at two measurement points before (pre, $n=376$ ) and during (peri, $n=640$ ) the COVID-19 pandemic in Poland, including 495 boys $(48.7 \%)$ and 521 girls (51.3\%). It was found that children in the pre-group were more physically active than children in the peri group $(p<0.0001)$. The average value $(\mathrm{Me})$ of the level of physical activity before the pandemic was 4 days with 60 min or longer physical activity, while during the pandemic, it dropped to 3 days. In addition, a recommendation for physical activity for children was met by $12.3 \%$ of children before the pandemic and $9.2 \%$ during the pandemic, but this difference was not significant $(p=0.1220)$.

Significant differences in the duration spent sleeping during a $24 \mathrm{~h}$ period were observed between the two groups $(p=0.0009)$. Both on school days and on weekends, a decrease in sleep time was noticed. Sleep time on weekdays dropped from 8.83 to $8.55 \mathrm{~h}$ and on the weekend from 10.11 to $9.52 \mathrm{~h}$. However, we found that the sleep quality was significantly higher during the pandemic $(p=0.0323)$.

The differences between the pre and peri groups are shown below (Table 1). 
Table 1. The pre- and peri groups differences.

\begin{tabular}{|c|c|c|c|c|c|c|c|c|}
\hline \multicolumn{3}{|l|}{ Variable } & $\bar{x}$ & SD & Me & Min & Max & $p$ \\
\hline \multirow{2}{*}{ Age (years) } & \multirow{2}{*}{\multicolumn{2}{|c|}{$\begin{array}{l}\text { pre } \\
\text { peri }\end{array}$}} & 10.51 & 2.13 & 10.00 & 6.00 & 15.00 & \multirow{2}{*}{0.1032} \\
\hline & & & 10.79 & 2.02 & 10.00 & 6.00 & 15.00 & \\
\hline \multirow{2}{*}{ Height (cm) } & \multirow{2}{*}{\multicolumn{2}{|c|}{$\begin{array}{l}\text { pre } \\
\text { peri }\end{array}$}} & 148.33 & 13.93 & 148.00 & 116.00 & 183.00 & \multirow{2}{*}{0.0255} \\
\hline & & & 146.48 & 13.93 & 146.00 & 110.00 & 197.00 & \\
\hline \multirow{2}{*}{ Weight (kg) } & \multirow{2}{*}{\multicolumn{2}{|c|}{$\begin{array}{l}\text { pre } \\
\text { peri }\end{array}$}} & 42.40 & 14.38 & 39.80 & 19.00 & 107.30 & \multirow{2}{*}{0.0536} \\
\hline & & & 40.58 & 13.36 & 38.00 & 19.00 & 97.00 & \\
\hline \multirow{2}{*}{$\mathrm{BMI}\left(\mathrm{kg} / \mathrm{m}^{2}\right)$} & \multirow{2}{*}{\multicolumn{2}{|c|}{$\begin{array}{l}\text { pre } \\
\text { peri }\end{array}$}} & 18.78 & 3.83 & 17.90 & 12.10 & 39.90 & \multirow{2}{*}{0.2099} \\
\hline & & & 18.46 & 3.58 & 17.78 & 11.83 & 34.67 & \\
\hline \multirow{2}{*}{$\begin{array}{l}\text { Days with } 60 \text { min or longer physical } \\
\text { activity }\end{array}$} & \multirow{2}{*}{\multicolumn{2}{|c|}{$\begin{array}{l}\text { pre } \\
\text { peri }\end{array}$}} & 3.89 & 1.89 & 4.00 & 0.00 & 7.00 & \multirow[b]{2}{*}{$<0.0001$} \\
\hline & & & 3.30 & 2.07 & 3.00 & 0.00 & 7.00 & \\
\hline \multirow{4}{*}{$\begin{array}{l}\text { Duration spent sleepingduring a } 24 \mathrm{~h} \\
\text { period }(\mathrm{h})\end{array}$} & pre & Weekdays & 8.83 & 1.64 & 9.00 & 4.00 & 13.45 & \multirow{2}{*}{0.0009} \\
\hline & peri & Weekdays & 8.55 & 1.17 & 9.00 & 3.00 & 12.00 & \\
\hline & pre & Weekend & 10.11 & 1.45 & 10.00 & 5.00 & 15.30 & \multirow{2}{*}{$<0.0001$} \\
\hline & peri & Weekend & 9.52 & 1.36 & 9.50 & 4.00 & 15.00 & \\
\hline \multirow{2}{*}{ Sleep quality } & \multirow{2}{*}{\multicolumn{2}{|c|}{$\begin{array}{l}\text { pre } \\
\text { peri }\end{array}$}} & 1.70 & 0.68 & 2.00 & 1.00 & 4.00 & \multirow{2}{*}{0.0323} \\
\hline & & & 1.78 & 0.65 & 2.00 & 1.00 & 4.00 & \\
\hline
\end{tabular}

$\bar{x}$-arithmetic mean, Me—median, SD—standard deviation, Min—sample minimum, Max—sample maximum, $p$ - $p$ value, indicate significant values $(p<0.05)$. Bold values denote statistical significance at the $\mathrm{p}<0.05$ level.

Figure 1 presents the body weight categories based on BMI centiles. There were no significant differences between the groups pre and peri COVID-19.

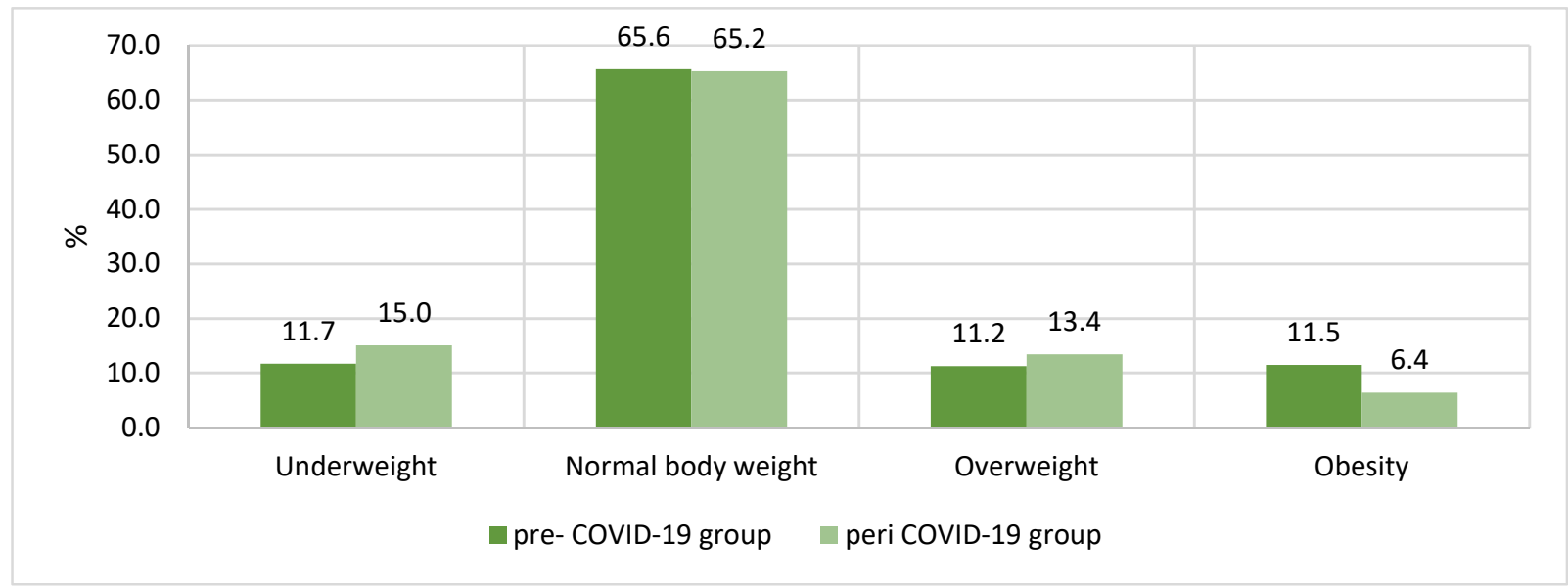

Figure 1. Body weight categories based on BMI centiles in the groups pre- and peri COVID-19 $(p=0.065)$.

Characteristics of media usage in the groups pre- and peri COVID-19 are presented in Table 2. Significant differences in time of watching movies or programs on the Internet or TV were found between children from the group before the pandemic and during the pandemic. During the pandemic, children often watched significantly more movies or programs on the Internet or TV on weekdays but not on the weekend. On the weekdays, the percentage of children who watched more than $6 \mathrm{~h}$ per day increased from $1.3 \%$ to $5.1 \%(p=0.0319)$. Moreover, children during the pandemic significantly more often spent time on the weekdays on playing games $(1.29 \mathrm{~h}$ vs. $1.64 \mathrm{~h} ; p<0.0001)$. However, using a smartphone on a regular day has decreased during the pandemic from 3.14 to 2.81 per day $(p=0.0016)$. 
Table 2. The media usage characteristics in the groups pre- and peri COVID-19.

\begin{tabular}{|c|c|c|c|c|c|c|c|c|c|c|c|}
\hline Variable & & & $\bar{x}$ & SD & NAA(\%) & $\begin{array}{l}<30 \\
\min \\
(\%)\end{array}$ & $\begin{array}{c}30 \\
\text { min-2 } \\
\mathrm{h}(\%)\end{array}$ & $\begin{array}{c}\approx 2-3 \\
\mathrm{~h}(\%)\end{array}$ & $\begin{array}{c}\approx 3-6 \\
\mathrm{~h}(\%)\end{array}$ & $>6 \mathrm{~h}(\%)$ & $p$ \\
\hline \multirow{4}{*}{$\begin{array}{c}\text { Time of watching movies } \\
\text { or programs on the } \\
\text { Internet (on an iPad, } \\
\text { tablet, computer, } \\
\text { smartphone) or TV per } \\
\text { day [h] }\end{array}$} & pre & Weekdays & 2.12 & 1.00 & 11.7 & 0.0 & 61.1 & 20.5 & 5.3 & 1.3 & \multirow[b]{2}{*}{0.0319} \\
\hline & peri & Weekdays & 2.34 & 1.12 & 4.4 & 14.0 & 44.0 & 23.4 & 9.0 & 5.1 & \\
\hline & pre & Weekend & 2.81 & 1.04 & 4.3 & 0.0 & 34.4 & 37.6 & 18.9 & 4.8 & \multirow[b]{2}{*}{0.0276} \\
\hline & peri & Weekend & 2.70 & 1.10 & 2.3 & 8.0 & 35.9 & 31.5 & 16.2 & 6.1 & \\
\hline \multirow{4}{*}{$\begin{array}{c}\text { Time of playing games } \\
\text { (on your computer/game } \\
\text { con- } \\
\text { sole/smartphone/iPad } \\
\text { etc.) per day [h] }\end{array}$} & pre & Weekdays & 1.29 & 1.08 & 27.7 & 30.1 & 31.5 & 7.2 & 2.7 & 0.8 & \multirow[b]{2}{*}{$<0.0001$} \\
\hline & peri & Weekdays & 1.64 & 1.23 & 20.3 & 27.8 & 29.5 & 14.7 & 5.9 & 1.9 & \\
\hline & pre & Weekend & 1.94 & 1.23 & 13.3 & 21.3 & 37.6 & 16.0 & 9.1 & 2.7 & \multirow{2}{*}{0.0599} \\
\hline & peri & Weekend & 2.11 & 1.29 & 11.4 & 20.4 & 34.2 & 18.6 & 11.1 & 4.4 & \\
\hline Variable & & $\bar{x}$ & SD & $\begin{array}{c}\text { I } \\
\text { Don't } \\
\text { Have } \\
\text { Access } \\
(\%)\end{array}$ & $\begin{array}{c}<5 \text { Times } \\
\text { a Day } \\
(\%)\end{array}$ & $\begin{array}{c}\text { 6-10 } \\
\text { Times } \\
\text { aDay } \\
(\%)\end{array}$ & $\begin{array}{c}\text { 11-20 } \\
\text { Timesa } \\
\text { Day } \\
(\%)\end{array}$ & $\begin{array}{c}21-50 \\
\text { Timesa } \\
\text { Day (\%) }\end{array}$ & $\begin{array}{c}51-100 \\
\text { Timesa } \\
\text { Day } \\
(\%)\end{array}$ & $\begin{array}{c}>100 \\
\text { TimesPer } \\
\text { Day }(\%)\end{array}$ & $p$ \\
\hline \multirow{2}{*}{$\begin{array}{l}\text { Using a smartphone on a } \\
\text { regular day }\end{array}$} & pre & 3.14 & 1.65 & 18.4 & 22.1 & 22.1 & 14.7 & 13.1 & 6.1 & 3.5 & \multirow{2}{*}{0.0016} \\
\hline & peri & 2.81 & 1.58 & 23.6 & 26.8 & 20.0 & 13.3 & 9.5 & 4.4 & 2.5 & \\
\hline
\end{tabular}

NAA—not at all, $\bar{x}$-arithmetic mean, SD—standard deviation, $p-p$ value, indicate significant values $(p<0.05)$. Bold values denote statistical significance at the $\mathrm{p}<0.05$ level.

In Table 3, the difference between the two groups in frequency of food consumption is shown. It was found that the frequency of consumption of most products has decreased $(\bar{x}$; pre vs. peri): legumes (1.63 vs. 1.36), potatoes ( 2.82 vs. 2.51$)$, fruit juices ( 3.28 vs. 2.77$)$, carbonated sugar sweetened drinks (1.77 vs. 1.40$)$, diet carbonated drinks (1.43 vs. 1.22$)$, cold cuts and preserved, ready-to-cook meat product (2.94 vs. 2.61$)$, fast food (1.76 vs. 1.39$)$, nuts and seeds ( 2.00 vs. 1.76), and snacks (1.95 vs. 1.56$)$.

Table 3. Differences in frequency of food intake between children before and during the pandemic.

\begin{tabular}{|c|c|c|c|c|c|c|c|c|c|c|c|}
\hline Selected Food Items & & $\bar{x}$ & SD & $\begin{array}{c}\text { Never/Less } \\
\text { than Once } \\
\text { a Week } \\
(\%)\end{array}$ & $\begin{array}{c}1-3 \\
\text { Times } \\
\text { a } \\
\text { Week } \\
(\%)\end{array}$ & $\begin{array}{c}4-6 \\
\text { Times } \\
\text { a } \\
\text { Week } \\
(\%)\end{array}$ & $\begin{array}{l}1 \text { Time } \\
\text { Per } \\
\text { Day } \\
(\%)\end{array}$ & $\begin{array}{l}2 \text { Times } \\
\text { Per Day } \\
\quad(\%)\end{array}$ & $\begin{array}{l}3 \text { Times } \\
\text { Per Day } \\
(\%)\end{array}$ & $\begin{array}{c}4 \text { or } \\
\text { More } \\
\text { Times } \\
\text { Per Day } \\
\text { (\%) }\end{array}$ & $p$ \\
\hline \multirow{2}{*}{$\begin{array}{l}\text { Legumes (e.g., beans, } \\
\text { lentils, chickpeas) }\end{array}$} & pre & 1.63 & 1.14 & 60.5 & 29.6 & 4.5 & 1.9 & 0.8 & 0.3 & 2.4 & \multirow[b]{2}{*}{0.0009} \\
\hline & peri & 1.36 & 0.65 & 68.8 & 28.7 & 1.6 & 0.3 & 0.2 & 0.2 & 0.3 & \\
\hline \multirow{2}{*}{ Potatoes (cooked) } & pre & 2.82 & 1.27 & 4.0 & 44.8 & 34.7 & 8.5 & 2.1 & 1.1 & 4.8 & \multirow{2}{*}{$<0.0001$} \\
\hline & peri & 2.51 & 1.01 & 5.8 & 55.1 & 29.3 & 6.4 & 0.6 & 0.9 & 1.9 & \\
\hline \multirow{2}{*}{$\begin{array}{c}\text { Raw vegetables (mixed } \\
\text { salad, carrot, fennel, } \\
\text { cucumber, lettuce, tomato) }\end{array}$} & pre & 2.90 & 1.57 & 16.8 & 29.3 & 30.1 & 9.6 & 4.8 & 4.0 & 5.3 & \multirow[b]{2}{*}{0.4869} \\
\hline & peri & 2.85 & 1.29 & 12.5 & 35.6 & 19.5 & 24.6 & 4.2 & 2.2 & 1.4 & \\
\hline \multirow[b]{2}{*}{ Fresh fruits } & pre & 3.55 & 1.77 & 8.8 & 24.0 & 25.9 & 12.5 & 12.3 & 6.1 & 10.4 & \multirow[b]{2}{*}{0.7831} \\
\hline & peri & 3.37 & 1.31 & 4.7 & 25.7 & 18.7 & 38.4 & 6.6 & 2.8 & 3.1 & \\
\hline \multirow{2}{*}{$\begin{array}{l}\text { Fruit juices (100\% fruit), } \\
\text { packaged }\end{array}$} & pre & 3.28 & 1.75 & 11.7 & 32.3 & 18.1 & 14.4 & 7.5 & 9.6 & 6.4 & \multirow{2}{*}{$<0.0001$} \\
\hline & peri & 2.77 & 1.42 & 16.1 & 36.8 & 19.2 & 18.1 & 4.2 & 2.8 & 2.8 & \\
\hline \multirow{2}{*}{$\begin{array}{c}\text { Carbonated sugar } \\
\text { sweetened drinks (e.g., coca } \\
\text { cola, Fanta) }\end{array}$} & pre & 1.77 & 1.22 & 56.8 & 27.2 & 7.2 & 3.5 & 2.9 & 1.1 & 1.3 & \multirow[b]{2}{*}{$<0.0001$} \\
\hline & peri & 1.40 & 0.81 & 71.1 & 22.8 & 3.4 & 1.7 & 0.0 & 0.3 & 0.6 & \\
\hline \multirow{2}{*}{$\begin{array}{l}\text { Diet carbonated drinks } \\
\text { (e.g., diet cola) }\end{array}$} & pre & 1.43 & 1.03 & 78.1 & 12.3 & 4.0 & 2.7 & 1.1 & 1.1 & 0.8 & \multirow{2}{*}{0.0176} \\
\hline & peri & 1.22 & 0.58 & 83.2 & 13.9 & 1.9 & 0.5 & 0.3 & 0.3 & 0 & \\
\hline \multirow{2}{*}{$\begin{array}{l}\text { Sweetened or sugar added } \\
\text { breakfast cereals }\end{array}$} & pre & 2.21 & 1.30 & 34.7 & 34.1 & 16.8 & 10.1 & 0.8 & 1.3 & 2.1 & \multirow{2}{*}{0.6000} \\
\hline & peri & 2.18 & 1.12 & 31.4 & 38.5 & 13.9 & 13.9 & 1.7 & 0.3 & 0.3 & \\
\hline
\end{tabular}


Table 3. Cont.

\begin{tabular}{|c|c|c|c|c|c|c|c|c|c|c|c|}
\hline Selected Food Items & & $\bar{x}$ & SD & $\begin{array}{l}\text { Never/Less } \\
\text { than Once } \\
\text { a Week } \\
(\%)\end{array}$ & $\begin{array}{c}1-3 \\
\text { Times } \\
\text { a } \\
\text { Week } \\
(\%)\end{array}$ & $\begin{array}{c}4-6 \\
\text { Times } \\
\text { a } \\
\text { Week } \\
(\%)\end{array}$ & $\begin{array}{c}1 \text { Time } \\
\text { Per } \\
\text { Day } \\
(\%)\end{array}$ & $\begin{array}{l}2 \text { Times } \\
\text { Per Day } \\
\text { (\%) }\end{array}$ & $\begin{array}{l}3 \text { Times } \\
\text { Per Day } \\
(\%)\end{array}$ & $\begin{array}{c}4 \text { or } \\
\text { More } \\
\text { Times } \\
\text { Per Day } \\
\text { (\%) }\end{array}$ & $p$ \\
\hline \multirow{2}{*}{ Plain unsweetened milk } & pre & 2.34 & 1.52 & 35.7 & 30.4 & 17.1 & 8.3 & 2.7 & 1.6 & 4.3 & \multirow{2}{*}{$<0.0001$} \\
\hline & peri & 2.83 & 1.22 & 14.8 & 28.4 & 22.9 & 29.3 & 2.7 & 0.8 & 1.1 & \\
\hline \multirow{2}{*}{$\begin{array}{c}\text { Plain unsweetened yoghurt } \\
\text { or kefir }\end{array}$} & pre & 1.71 & 1.11 & 57.1 & 28.5 & 6.9 & 4.8 & 0.3 & 1.6 & 0.8 & \multirow{2}{*}{0.0009} \\
\hline & peri & 1.80 & 0.89 & 44.3 & 39.2 & 9.8 & 6.1 & 0.6 & 0 & 0 & \\
\hline \multirow{2}{*}{ Sweet and flavored yoghurt } & pre & 2.15 & 1.24 & 32.8 & 40.0 & 16.3 & 6.1 & 1.6 & 1.3 & 1.9 & \multirow{2}{*}{0.0147} \\
\hline & peri & 2.23 & 1.03 & 23.9 & 45.4 & 17.5 & 11.5 & 0.9 & 0.5 & 0.3 & \\
\hline \multirow{2}{*}{$\begin{array}{l}\text { Fish, boiled, grilled, oven } \\
\text { baked, raw }\end{array}$} & pre & 1.52 & 0.90 & 61.6 & 32.3 & 2.4 & 2.1 & 0.5 & 0 & 1.1 & \multirow{2}{*}{0.0058} \\
\hline & peri & 1.55 & 0.67 & 50.7 & 46.0 & 2.2 & 0.5 & 0.3 & 0 & 0.3 & \\
\hline \multirow{2}{*}{ Fish, fried and/or coated } & pre & 1.55 & 0.86 & 58.1 & 34.9 & 3.5 & 2.4 & 0.3 & 0 & 0.8 & \multirow{2}{*}{0.6061} \\
\hline & peri & 1.49 & 0.60 & 54.3 & 43.2 & 1.7 & 0.3 & 0.5 & 0 & 0 & \\
\hline \multirow{2}{*}{$\begin{array}{l}\text { Cold cuts and preserved, } \\
\text { ready-to-cook meat product }\end{array}$} & pre & 2.94 & 1.51 & 10.9 & 37.9 & 25.1 & 11.2 & 7.2 & 2.4 & 5.3 & \multirow{2}{*}{0.0272} \\
\hline & peri & 2.61 & 1.09 & 13.6 & 37.6 & 28.5 & 16.7 & 2.2 & 0.8 & 0.6 & \\
\hline \multirow{2}{*}{$\begin{array}{l}\text { Poultry, meat, boiled, } \\
\text { grilled, oven baked, } \\
\text { without coating not fried }\end{array}$} & pre & 1.98 & 1.17 & 37.6 & 43.2 & 12.0 & 2.1 & 1.9 & 2.1 & 1.1 & \multirow[b]{2}{*}{$<0.0001$} \\
\hline & peri & 2.27 & 0.85 & 13.6 & 54.6 & 26.2 & 4.2 & 0.6 & 0.3 & 0.5 & \\
\hline \multirow{2}{*}{ Fried poultry, meat } & pre & 1.98 & 1.18 & 36.8 & 46.4 & 8.5 & 3.5 & 2.1 & 0.8 & 1.9 & \multirow{2}{*}{0.0662} \\
\hline & peri & 1.71 & 0.59 & 35.4 & 58.7 & 5.5 & 0.5 & 0 & 0 & 0 & \\
\hline \multirow{2}{*}{$\begin{array}{l}\text { White bread, white roll, } \\
\text { white crispbread }\end{array}$} & pre & 3.30 & 1.67 & 10.9 & 27.5 & 25.6 & 10.9 & 13.9 & 4.3 & 6.9 & \multirow{2}{*}{0.0092} \\
\hline & peri & 3.35 & 1.12 & 6.1 & 17.8 & 22.5 & 46.2 & 4.5 & 2.2 & 0.8 & \\
\hline \multirow{2}{*}{$\begin{array}{l}\text { Whole meal bread, dark } \\
\text { roll, dark crispbread }\end{array}$} & pre & 2.43 & 1.38 & 25.3 & 39.2 & 18.4 & 8.5 & 4.0 & 2.1 & 2.4 & \multirow{2}{*}{0.0617} \\
\hline & peri & 2.22 & 1.17 & 31.8 & 35.1 & 16.7 & 13.3 & 1.7 & 1.2 & 0.2 & \\
\hline \multirow{2}{*}{$\begin{array}{l}\text { Pasta, noodles, rice and } \\
\text { other cereals, white, refined }\end{array}$} & pre & 2.18 & 1.22 & 25.6 & 51.5 & 14.1 & 2.9 & 2.7 & 0.5 & 2.7 & \multirow{2}{*}{0.0011} \\
\hline & peri & 2.19 & 0.73 & 10.8 & 65.5 & 19.0 & 3.9 & 0.3 & 0.3 & 0.2 & \\
\hline \multirow{2}{*}{$\begin{array}{l}\text { Whole meal pasta, noodles, } \\
\text { brown rice and other } \\
\text { cereals, unrefined }\end{array}$} & pre & 1.66 & 1.05 & 58.4 & 28.5 & 7.2 & 2.4 & 2.4 & 0.3 & 0.8 & \multirow{2}{*}{0.8144} \\
\hline & peri & 1.57 & 0.77 & 55.2 & 35.7 & 7.2 & 1.2 & 0.2 & 0.3 & 0.2 & \\
\hline \multirow{2}{*}{$\begin{array}{l}\text { Not homemade hamburger, } \\
\text { hot dog, kebab, wrap, } \\
\text { falafel, sandwich }\end{array}$} & pre & 1.76 & 1.29 & 58.1 & 26.9 & 6.7 & 2.9 & 1.9 & 1.1 & 2.4 & \multirow[b]{2}{*}{$<0.0001$} \\
\hline & peri & 1.39 & 0.68 & 69.0 & 25.3 & 4.7 & 0.8 & 0 & 0.2 & 0.2 & \\
\hline \multirow{2}{*}{ Nuts and seeds } & pre & 2.00 & 1.24 & 39.5 & 40.3 & 11.7 & 3.7 & 1.3 & 1.3 & 2.1 & م00- \\
\hline & peri & 1.76 & 0.90 & 45.2 & 41.3 & 8.0 & 4.5 & 0.3 & 0.3 & 0.3 & 0.0095 \\
\hline Snacks such as crisps, corn & pre & 1.95 & 1.30 & 45.3 & 35.5 & 9.3 & 4.5 & 1.6 & 1.3 & 2.4 & \\
\hline crisps, popcorn, etc. & peri & 1.56 & 0.65 & 51.2 & 42.9 & 4.8 & 0.8 & 0.3 & 0 & 0 & 0.0003 \\
\hline Snacks such as biscuits, & pre & 2.17 & 1.23 & 29.9 & 44.0 & 16.5 & 4.5 & 1.3 & 1.9 & 1.9 & \\
\hline $\begin{array}{l}\text { and puddings } \\
\text { packages }\end{array}$ & peri & 2.20 & 0.97 & 21.7 & 50.1 & 17.0 & 9.5 & 1.2 & 0.2 & 0.3 & 0.0371 \\
\hline
\end{tabular}

However, especially in milk products consumption, an increase has been observed $(p<0.05)$. Additionally, the consumption of boiled, grilled, oven baked, raw fish, poultry, and meat also increased $(p<0.05)$. It was also noticed that the consumption of sweets increased $(p=0.0371)$.

\section{Discussion}

Our results present that there were differences in eating behaviors, level of physical activity, hours of sleep, and screen time among Polish children and adolescents aged 615 years before and during the COVID-19 pandemic. To our knowledge, this is the first study conducted in Poland among children before and during the COVID-19 pandemic and assessing the change in media usage, sleep time, eating habits, and physical activity.

Our results show a decline in PA among children aged 6-15 years during the pandemic in contrast to the time before the lockdown. The average number of days with $60 \mathrm{~min}$ or longer physical activity dropped from 3.89 to 3.30. Data from the literature investigating 
changes in children's PA are limited. The first preliminary results indicate a downward trend in PA levels $[12,19]$. The main reason is probably social restriction such as remote learning. In addition, parental limitations due to 'shelter-at-home' recommendations and working from home may create difficulties in keeping children physically active. In a study of 1472 Canadian youth, only $3.6 \%$ of children aged $5-11$ and $2.6 \%$ aged $12-17$ years met the recommendation of achieving $60 \mathrm{~min}$ of moderate-vigorous physical activity/per day during the COVID-19 pandemic [12]. In our study, this recommendation was met by $9.2 \%$ of children (in contrast to the $12.3 \%$ before pandemic). In a study of 97 South Korean parents, $94 \%$ described a reduction in daily physical activity of their children during the COVID-19 pandemic [29].

Our study demonstrated the change in the sleep duration between the period before and during COVID-19 pandemic. Lack of sleep may have an impact on emotional health, attention span, and immune function [18,30]. Insufficient sleep can increase the risk of cardiometabolic disease in both children and adolescents and results in mood swings anxiety, which may be intensified by poor mental health during the COVID-19 pandemic $[30,35]$. The Australian Department of Health recommends between 9 and $11 \mathrm{~h}$ of sleep for children aged 5-13 years and 8-10 h of sleep for adolescents aged 14-17 years [36]. Our results show the decrease in the sleep duration both on the weekday and on the weekend. The average duration spent sleeping (including naps) was $8.83 \mathrm{~h}$ before and $8.55 \mathrm{~h}$ during the pandemic on the schooldays. On the weekend, the average duration spent sleeping also decreased from 10.11 to $9.52 \mathrm{~h}$. However, the sleep quality slightly increased. The decrease of the sleep duration can be explained by unscheduled sleep during the COVID19 pandemic, usually without parents' control. Children spent more time in front of the computer/tablet/television and extensive use of electronic devices was noticed; therefore, it could cause a delay in bedtime and as a consequence shortened sleep duration. In Poland, the distance learning was according to the school lessons plan, and the lessons were usually held from 8.00 a.m. Moore et al. showed that children were sleeping more hours than they had been prior to the COVID-19 pandemic [12]. Likewise, Pietrobelli et al. noticed that sleep increased by $0.65 \mathrm{~h}$ per day during the pandemic among Italian children compared to the period before the pandemic [13]. This difference was very small, but changes between our results and other studies can be explained by the specific sleeping and waking up routines among countries. In Poland, parents usually get up very early to work (both remotely and traditionally), which is why they often wake their children. However, data on sleep duration are inconsistent across the literature. Israelian research on infants and children presented inconsistent changes in sleep duration, both an increase and decrease or no changes [37].

Digital media and devices are an integral part of the modern world. During the pandemic, the use of these devices has become a condition of everyday functioning not only of adults but also of children [38]. Despite the potential benefits, excessive and inappropriate use of technology may have an effect on children's development and health. According to the previous research, there is a link between increased screen time and a greater risk of health complications, mental health issues, and the negative effects of cognitive, linguistic, social, and emotional development [39-41]. Our results showed significant differences in the time spent by children using the Internet and TV between groups before and during the pandemic. Moreover, the increase in time in front of the monitor mainly concerned days during the week, not on the weekend, as it was before the pandemic. The percentage of children who spent more than $6 \mathrm{~h}$ per day watching TV and using the Internet increased from $1.3 \%$ to $5.1 \%(p=0.0319)$. The amount of time spent on computer games also increased $(1.29 \mathrm{~h}$ vs. $1.64 \mathrm{~h} ; p<0.0001)$, and this relates to weekdays. However, using a smartphone on a regular day has decreased during the pandemic from 3.14 to 2.81 per day $(p=0.0016)$. According to the American Academy of Pediatrics report from 2019, even before the pandemic, children spent a lot of time in front of the screen; children aged 8-12 spent an average of $4.5 \mathrm{~h}$ a day on screens, while teens aged $13-18$ spent $6.5 \mathrm{~h}$ a day $[42,43]$. The pandemic and the resulting closures of schools, 
sports, and most extra-curricular activities only increased our dependence on digital tools, thereby increasing the children's time spent in front of the monitor. As reported by the Statista report, one of the largest statistical websites in the world, in the USA, the amount of screen time spent by children of all age groups increased by an average of $4 \mathrm{~h}$ a day. The pandemic has increased the use of electronic devices among American children and teens, whose screen time is now twice as high as it used to be [44]. Similar results were obtained in a German study of 1717 participants at two measurement points before and during the pandemic, which was published in the journal Nature [45]. Schmidt et al. showed that watching TV and recreational Internet browsing were significant factors that influenced the extension of children's time in front of the monitor [45]. Our results are also consistent with an experimental longitudinal study conducted among children and adolescents in China [14] and in Spain [45]. It shows that the special circumstances during COVID-19 have caused many changes in children's media and electronic device use habits. It is extremely important to use the available evidence and prevent unhealthy screen time that may affect health and well-being among children.

Our study found that the restrictions as a result of the COVID-19 pandemic induced reductions in the consumption of certain foods such as legumes, potatoes, fruit juices, carbonated sugar, sweetened drinks, diet sodas, meats and canned food, ready-to-cook meat products, fast food, nuts and seeds, and snacks. There was an increase in the consumption of dairy products. Additionally, the consumption of cooked, grilled, baked in the oven, raw fish, poultry, and meat as well as sweets increased. The consumption of fresh vegetables and fruit was lower, but no statistically significant differences were observed. A Spanish study found significant reductions in fruit and vegetable consumption during the COVID19 pandemic in a subset of children aged 3 to 5 years. In the group of children aged 6-16, it was also smaller, but no statistically significant differences were observed [46]. Similarly, a study with Italian children and adolescents whose schools closed due to the COVID-19 pandemic showed a higher consumption of red meat and potato chips [13]. General eating habits may have deteriorated during the pandemic. In addition, less food consumption outside the home during the pandemic has been associated with changes in dietary quality, including lower consumption of fast food. On the one hand, restrictions resulting from leaving the house only to perform permitted activities could have resulted in limited access to, for example, fresh fruit and vegetables. However, staying home and closing businesses may have reduced exposure to processed foods such as fast-food restaurants.

There were a number of potential limitations present in this study that should be considered when interpreting the results. Self-submitted and parental questionnaires were used to assess children's eating habits, physical activity, sleep, and media use. Selfreported data are likely to recall bias and social desirability. Additionally, it was the cross-sectional study from two different measurement points. It would be a more helpful and meaningful study using the within-subjects method rather than between subjects. Repeated measurements (pre/peri COVID-19 groups) strengthened the study but did not allow for a population-specific generalization of the changes in that period. The limitation is also the lack of details about the intensity of PA.

\section{Conclusions}

In conclusion, our study comprehensively examined eating habits, physical activity, sleep, and media usage differences between children before the COVID-19 pandemic and one year later during the pandemic. The study identified dietary differences (e.g., reductions in the consumption of fruit juices, carbonated sugar, sweetened drinks, diet sodas, meats and canned food, fast food, snacks and increase in the consumption of dairy products, fish, poultry, meat, and sweets) and changes in daily activity patterns (reduced sleep duration with higher sleep quality and reduced physical activity). Additionally, an increase in general media usage was observed during the pandemic alongside a decrease in smartphone usage. The obtained results indicate increased sleep, physical activity, and 
reduced media usage and screen time among Polish children and adolescents during the COVID-19 pandemic.

Author Contributions: Conceptualization, E.Ł. and M.K.; Data curation, P.J., Ł.O. and A.S.; Formal analysis, P.J. and Ł.O.; Investigation, A.B., I.P.-Ś., M.K. and K.D.; Methodology, E.Ł., A.B. and I.P.-Ś.; Resources, A.S. and K.D.; Writing—original draft, E.E., A.B. and K.D.; Writing—review and editing, E.E. All authors have read and agreed to the published version of the manuscript.

Funding: This research received no external funding.

Institutional Review Board Statement: This research project was carried out in accordance with the Helsinki Declaration. The study was approved by the institutional Bioethics Committee at the University of Rzeszów (Resolution No. 4/05/2021) and all appropriate administrative bodies.

Informed Consent Statement: Informed consent was obtained from all subjects involved in the study.

Data Availability Statement: The data presented in this study are available on reasonable request from the corresponding author. The data are not publicly available due to restrictions e.g., their containing information that could compromise the privacy of research participants.

Conflicts of Interest: The authors declare no conflict of interest.

\section{References}

1. Bonaccorsi, G.; Pierri, F.; Cinelli, M.; Flori, A.; Galeazzi, A.; Porcelli, F.; Schmidt, A.L.; Valensise, C.M.; Scala, A.; Quattrociocchi, W.; et al. Economic and social consequences of human mobility restrictions under COVID-19. Proc. Natl. Acad. Sci. USA 2020, 117, 15530-15535. [CrossRef]

2. L'Angiocola, P.D.; Monti, M. COVID-19: The critical balance between appropriate governmental restrictions and expected economic, psychological and social consequences in Italy. Are we going in the right direction? Acta Biomed. Atenei Parm. 2020, 91, 35-38.

3. Morgül, E.; Kallitsoglou, A.; Essau, C.A. Psychological effects of the COVID-19 lockdown on children and families in the UK. Rev. Psicol. Clín. Niños Adolesc. 2020, 7, 7. [CrossRef]

4. Singh, S.; Roy, D.; Sinha, K.; Parveen, S.; Sharma, G.; Joshi, G. Impact of COVID-19 and lockdown on mental health of children and adolescents: A narrative review with recommendations. Psychiatry Res. 2020, 293, 113429. [CrossRef]

5. Guthold, R.; Stevens, G.A.; Riley, L.M.; Bull, F.C. Global trends in insufficient physical activity among adolescents: A pooled analysis of 298 population-based surveys with 1.6 million participants. Lancet Child Adolesc. Health 2020, 4, 23-35. [CrossRef]

6. Chtourou, H.; Trabelsi, K.; H'Mida, C.; Boukhris, O.; Glenn, J.M.; Brach, M.; Bentlage, E.; Bott, N.; Shephard, R.J.; Ammar, A.; et al. Staying Physically Active During the Quarantine and Self-Isolation Period for Controlling and Mitigating the COVID-19 Pandemic: A Systematic Overview of the Literature. Front. Psychol. 2020, 11, 1708. [CrossRef]

7. Fazeli, S.; Zeidi, I.M.; Lin, C.-Y.; Namdar, P.; Griffiths, M.D.; Ahorsu, D.K.; Pakpour, A.H. Depression, anxiety, and stress mediate the associations between internet gaming disorder, insomnia, and quality of life during the COVID-19 outbreak. Addict. Behav. Rep. 2020, 12, 100307. [CrossRef] [PubMed]

8. Nobari, H.; Fashi, M.; Eskandari, A.; Villafaina, S.; Murillo-Garcia, Á.; Pérez-Gómez, J. Effect of COVID-19 on Health-Related Quality of Life in Adolescents and Children: A Systematic Review. Int. J. Environ. Res. Public Health 2021, 18, 4563. [CrossRef] [PubMed]

9. UNESCO. COVID-19 Educational Disruption and Response. 2020. Available online: https://en.unesco.org/news/covid-19 -educational-disruption-andresponse (accessed on 1 March 2021).

10. Bull, F.C.; Al-Ansari, S.S.; Biddle, S.; Borodulin, K.; Buman, M.P.; Cardon, G. World Health Organization 2020 guide-lines on physical activity and sedentary behaviour. Br. J. Sports Med. 2020, 54, 1451-1462. [CrossRef]

11. Dunton, G.F.; Do, B.; Wang, S.D. Early effects of the COVID-19 pandemic on physical activity and sedentary behavior in children living in the U.S. BMC Public Health 2020, 20, 1-13. [CrossRef]

12. Moore, S.; Faulkner, G.; Rhodes, R.; Brussoni, M.; Chulak-Bozzer, T.; Ferguson, L.; Mitra, R.; O’Reilly, N.; Spence, J.; Vanderloo, L.; et al. Impact of the COVID-19 virus outbreak on movement and play behaviours of Canadian children and youth: A national survey. Int. J. Behav. Nutr. Phys. Act. 2020, 17, 1-11. [CrossRef] [PubMed]

13. Pietrobelli, A.; Pecoraro, L.; Ferruzzi, A.; Heo, M.; Faith, M.; Zoller, T.; Antoniazzi, F.; Piacentini, G.; Fearnbach, S.N.; Heymsfield, S.B. Effects of COVID-19 Lockdown on Lifestyle Behaviors in Children with Obesity Living in Verona. Italy: A Longitudinal Study. Obesity 2020, 28, 1382-1385. [CrossRef] [PubMed]

14. Xiang, M.; Zhang, Z.; Kuwahara, K. Impact of COVID-19 pandemic on children and adolescents' lifestyle behavior larger than expected. Prog. Cardiovasc. Dis. 2020, 63, 531-532. [CrossRef] [PubMed] 
15. Roman-Viñas, B.; Chaput, J.P.; Katzmarzyk, P.T.; Fogelholm, M.; Lambert, E.V.; Maher, C.; Maia, J.; Olds, T.; Onywera, V.; Sarmiento, O.L.; et al. Proportion of children meeting recommendations for 24-h movement guidelines and associations with adiposity in a 12-country study. Int. J. Behav. Nutr. Phys. Act. 2016, 13, 123. [CrossRef]

16. Regulation of the Minister of Health of 20 March. Introducing a Nationwide State of Epidemic on the Territory of the Republic of Poland. Available online: https:/ /isap.sejm.gov.pl/isap.nsf/download.xsp/WDU20200000491/O/D20200491.pdf (accessed on 2 June 2021).

17. Margaritis, I.; Houdart, S.; El Ouadrhiri, Y.; Bigard, X.; Vuillemin, A.; Duché, P. How to deal with COVID-19 epidem-ic-related lockdown physical inactivity and sedentary increase in youth? Adaptation of Anses' benchmarks. Arch. Public Health 2020, 78, 1-6. [CrossRef]

18. Becker, S.P.; Gregory, A.M. Editorial Perspective: Perils and promise for child and adolescent sleep and associated psychopathology during the COVID-19 pandemic. J. Child. Psychol. Psychiatry Allied Discip. 2020, 61, 757-759. [CrossRef]

19. Zenic, N.; Taiar, R.; Gilic, B.; Blazevic, M.; Maric, D.; Pojskic, H.; Sekulic, D. Levels and changes of physical activity in ado-lescents during the COVID-19 Pandemic: Contextualizing urban vs. Rural living environment. Appl. Sci. 2020, 10, 3997. [CrossRef]

20. Fore, H.H. A wake-up call: COVID-19 and its impact on children's health and wellbeing. Lancet Glob. Health 2020, 8, e861-e862. [CrossRef]

21. El-Shabrawi, M.; Hassanin, F. Infant and child health and healthcare before and after COVID-19 pandemic: Will it be the same ever? Egypt. Pediatr. Assoc. Gaz. 2020, 68, 1-6. [CrossRef]

22. Mayurasakorn, K.; Pinsawas, B.; Mongkolsucharitkul, P.; Sranacharoenpong, K.; Damapong, S.-N. School closure, COVID-19 and lunch programme: Unprecedented undernutrition crisis in low-middle income countries. J. Paediatr. Child Health 2020, 56, 1013-1017. [CrossRef] [PubMed]

23. Akseer, N.; Kandru, G.; Keats, E.C.; Bhutta, Z.A. COVID-19 pandemic and mitigation strategies: Implications for maternal and child health and nutrition. Am. J. Clin. Nutr. 2020, 112, 251-256. [CrossRef]

24. Sidor, A.; Rzymski, P. Dietary Choices and Habits during COVID-19 Lockdown: Experience from Poland. Nutrients 2020, $12,1657$. [CrossRef] [PubMed]

25. Carson, V.; Hunter, S.; Kuzik, N.; Gray, C.E.; Poitras, V.J.; Chaput, J.-P.; Saunders, T.J.; Katzmarzyk, P.T.; Okely, A.D.; Gorber, S.C.; et al. Systematic review of sedentary behaviour and health indicators in school-aged children and youth: An update. Appl. Physiol. Nutr. Metab. 2016, 41, S240-S265. [CrossRef]

26. Tremblay, M.S.; Carson, V.; Chaput, J.-P.; Connor Gorber, S.; Dinh, T.; Duggan, M.; Faulkner, G.; Gray, C.E.; Gruber, R.; Janson, K.; et al. Canadian 24-Hour Movement Guidelines for Children and Youth: An Integration of Physical Activity. Sed-entary Behaviour. and Sleep. Appl. Physiol. Nutr. Metab. 2016, 41, S311-S327. [CrossRef] [PubMed]

27. Fakhouri, T.H.I.; Hughes, J.P.; Brody, D.J.; Kit, B.K.; Ogden, C.L. Physical Activity and Screen-Time Viewing Among Ele-mentary School-Aged Children in the United States From 2009 to 2010. JAMA Pediatr. 2013, 167, 223-229. [CrossRef] [PubMed]

28. Łuszczki, E.; Bartosiewicz, A.; Bobula, G.; Kuchciak, M.; Jagielski, P.; Oleksy, Ł.; Stolarczyk, A.; Dereń, K. New Media Development, Sleep and Lifestyle in Children and Adolescents. Sustainability 2021, 13, 2248. [CrossRef]

29. Guan, H.; Okely, A.D.; Aguilar-Farias, N.; Cruz, B.D.P.; Draper, C.E.; El Hamdouchi, A.; Florindo, A.A.; Jáuregui, A.; Katzmarzyk, P.T.; Kontsevaya, A.; et al. Promoting healthy movement behaviours among children during the COVID-19 pandemic. Lancet Child Adolesc. Health 2020, 4, 416-418. [CrossRef]

30. Paruthi, S.; Brooks, L.J.; Ambrosio, C.D.; Hall, W.A.; Kotagal, S.; Lloyd, R.M.; Malow, B.A.; Maski, K.; Nichols, C.; Quan, S.F.; et al. Consensus Statement of the American Academy of Sleep Medicine. J. Clin. Sleep Med. 2016, 12, 1553-1561.

31. Bartosiewicz, A.; Łuszczki, E.; Kuchciak, M.; Bobula, G.; Oleksy, Ł.; Stolarczyk, A.; Dereń, K. Children's Body Mass Index Depending on Dietary Patterns, the Use of Technological Devices, the Internet and Sleep on BMI in Children. Int. J. Environ. Res. Public Health 2020, 17, 7492. [CrossRef]

32. Kułaga, Z.; Różańska, A.; Palczewska, I. Percentile charts of height, body mass and body mass index in children and adoles-cents in Poland-Results of the OLAF study. Stand Med. 2010, 7, 690-700.

33. Wadołowska, L. Validation of food frequency questionnaire [FFQ]. Reproducibility assessment. Bromatol. Chem. Toksykol. 2005, 38, 27-33.

34. Rideout, V.J.; Foehr, U.G.; Roberts, D.F. Generation M2: Media in the Lives of 8-to 18-Year-Oids; Kaiser Family Foundation: Washington, DC, USA, 2010.

35. Jiao, W.Y.; Wang, L.N.; Liu, J.; Fang, S.F.; Jiao, F.Y.; Pettobello-Mantovani, M.; Somekh, E. Behavioral and Emotional Disor-ders in Children during the COVID-19 Epidemic. J. Pediatr. 2020, 21, 264. [CrossRef] [PubMed]

36. Hirshkowitz, M.; Whiton, K.; Albert, S.M.; Alessi, C.; Bruni, O.; DonCarlos, L.; Hazen, N.; Herman, J.; Katz, E.S.; KheirandishGozal, L.; et al. National Sleep Foundation's sleep time duration recommendations: Methodology and results summary. Sleep Health 2015, 1, 40-43. [CrossRef] [PubMed]

37. Zreik, G.; Asraf, K.; Haimov, I.; Tikotzky, L. Maternal perceptions of sleep problems among children and mothers during the coronavirus disease 2019 (COVID-19) pandemic in Israel. J. Sleep Res. 2021, 30. [CrossRef]

38. Halla, G.; Laddu, D.R.; Phillips, S.A.; Lavie, C.J.; Arena, R. A tale of two pandemics: How will COVID-19 and global trends in physical inactivity and sedentary behavior affect one another? Prog. Cardiovasc. Dis. 2021, 64, 108-110. [CrossRef]

39. Korczak, D.J.; Madigan, S.; Colasanto, M. Children's physical activity and depression: A meta-analysis. Pediatrics 2017, 139, e20162266. [CrossRef] 
40. Haapala, E.A.; Vaisto, J.; Lintu, N. Physical activity and sedentary time in relation to academic achievement in children. J. Sci. Med. Sport 2017, 20, 583-589. [CrossRef]

41. Stiglic, N.; Viner, R.M. Effects of screentime on the health and well-being of children and adolescents: A systematic review of reviews. BMJ Open 2019, 9, e023191. [CrossRef]

42. American Academy of Pediatrics. Caring for Our Children, National Health and Safety Performance Standards. In American Academy of Pediatrics; American Public Health Association: Washington, DC, USA, 2019.

43. Ashton, J.; Beattie, R.M. Screen time in children and adolescents: Is there evidence to guide parents and policy? Lancet Child Adolesc. Health 2019, 3, 292-294. [CrossRef]

44. Johnson, J. Children and Teens from the United States Who Spent More Than Four Hours Daily Using Electronics Devices Before and During the Coronavirus Pandemic according to Parents as of June 2020, by Age Group. Statista 2021. Available online: https:/ / www.statista.com/statistics/1189204/us-teens-children-screen-time-daily-coronavirus-before-during (accessed on 7 June 2021).

45. Schmidt, S.C.E.; Anedda, B.; Burchartz, A.; Eichsteller, A.; Kolb, S.; Nigg, C.; Niessner, C.; Oriwol, D.; Worth, A.; Woll, A. Physical activity and screen time of children and adolescents before and during the COVID-19 lockdown in Germany: A natural experiment. Sci. Rep. 2020, 10, 1-12. [CrossRef]

46. López-Bueno, R.; López-Sánchez, G.F.; Casajús, J.A.; Calatayud, J.; Gil-Salmerón, A.; Grabovac, I.; Tully, M.A.; Smith, L. HealthRelated Behaviors Among School-Aged Children and Adolescents During the Spanish Covid-19 Confinement. Front. Pediatr. 2020, 8, 573. [CrossRef] [PubMed] 